\title{
The energy transfer in W UMa binary stars
}

\author{
Dengkai Jiang ${ }^{1,2}$, Jiangcheng Wang ${ }^{1}$,Zhanwen Han ${ }^{1}$ \\ Tianyu Jiang ${ }^{1,2}$, and Lifang $\mathbf{L i}^{1}$ \\ ${ }^{1}$ National Astronomical Observatories/Yunnan Observatory, Chinese Academy of Sciences, \\ Kunming 650011, China \\ email: jiangdengkai@hotmail.com \\ ${ }^{2}$ Graduate University of Chinese Academy Sciences, Beijing 100039, China
}

\begin{abstract}
The properties of W UMa binary stars are studied based on the well-determined physical parameters of $132 \mathrm{~W}$ UMa systems. It is found that the energy transfer rate has a maximum value at $\mathrm{q} \sim 0.58$. The relation between the energy transfer rate and the temperature deviation is also investigated, and the temperature of the secondary component is related to the energy transfer rate.
\end{abstract}

Keywords. stars: evolution-(stars:) binaries: close-(stars:) binaries: general

\section{Introduction}

W UMa binary stars are very common eclipsing variables in which both components are overflowing their inner Roche lobe surfaces. The surface temperatures of two components of W UMa binary stars are nearly equal, though the masses of two components are often greatly different. The over-luminosity of the secondaries is the result of the energy transfer between the two components within a convective envelope (Lucy 1968). Mochnacki (1981) suggested that the energy transfer rate of W UMa binary star depends on the mass ratio. Li, Han \& Zhang (2004) discussed the region of energy transfer in the common envelope of W UMa binary stars, and showed that the energy transfer may take place in the radiative region of common envelope. On the base of the well-determined physical parameters of 132 W UMa binary stars collected from the literature, the energy transfer of W UMa binary stars has been analyzed.

\section{The outcome of W UMa binary stars}

The energy transfer rate can be calculated by

$$
U=\frac{s t^{4}-q^{\alpha}}{1+q^{\alpha}}
$$

where s $=S_{2} / S_{1}, \mathrm{t}=T_{2 e} / T_{1 e} ; S_{1,2}, T_{1,2 e}$ are the surface area and the effective temperature of the components, respectively (Mochnacki 1981). The distribution of the energy transfer rate vs the mass ratio of W UMa binary stars is shown in the left panel of Figure 1. It is seen that the energy transfer rate of W-types is greater than that of A-types ( $\mathrm{Li}$ et al 2008). The energy transfer rate of W UMa binary stars in fact increases with increasing mass ratio if $q \sim 0.6$ as Wang (1994). But when the $q$ is greater than this value, the energy transfer rate is decreased quickly. We can obtain a polynomial relation between the energy transfer rate and mass ratio by the least-squares fitting as the following,

$$
U=-3.634 q^{3}+2.899 q^{2}+0.309 q+0.0769
$$



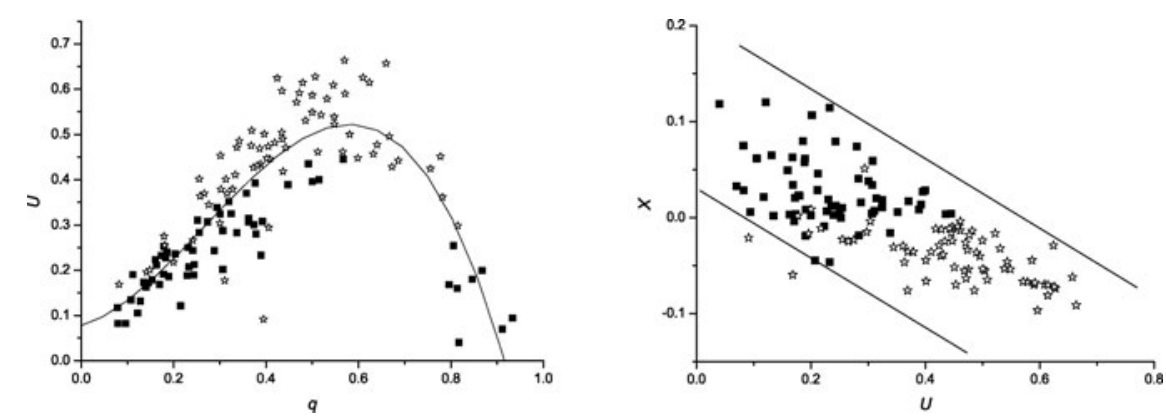

Figure 1. Left panel: The energy transfer rate as a function of mass ratio of W UMa binary stars. The solid line represents the Polynomial fit. Right panel: Relation of the energy transfer rate and the temperature deviation. Full squares:A-types; open stars:W-types.

according to equation (2.2), the energy transfer rate of W UMa binary stars peaks at a mass ratio of about 0.581 .

The temperature deviation is defined as

$$
\chi=\frac{T_{1 \mathrm{e}}-T_{2 \mathrm{e}}}{T_{2 \mathrm{e}}}
$$

where $T_{1 \mathrm{e}}$ and $T_{2 \mathrm{e}}$ are the effective temperatures of the primary and the secondary, respectively. The relation between the energy transfer rate and the temperature deviation is shown in the right panel of Figure 1. The W UMa binary stars seem to load in a strip limited by two solid lines, and there is a tendency for a decreasing energy transfer rate with increasing temperature deviation. It is clear that the temperature deviation is related to energy transfer rate. This indicates that the temperature of the secondary component increases with increasing energy transfer rate, or even exceed the temperature of the primary after the energy transfer rate is high enough.

\section{Summary}

We present the energy transfer of W UMa binary stars, and show the relation between the energy transfer rate and the mass ratio, together with the relation between the energy transfer rate and the relative temperature deviation. This study indicates that there is a peak of the energy transfer rate at $q=0.58$. The temperature of the secondary component is correlated with the energy transfer rate.

\section{Acknowledgements}

This work was partly supported by the Chinese Natural Science Foundation (10673029, 10773026, 10433030 and 10521001), and by the Yunnan Natural Science Foundation (2007A113M and 2005A0035Q).

\section{References}

Li, L., Zhang F., \& Han Z., 2004, MNRAS, 351, 137

Li, L., Zhang, F., Han, Z., Jiang, D., \& Jiang, T., 2008, MNRAS, inpress

Lucy, L. B., 1968, ApJ, 151, 1123

Mochnacki S., 1981, A\&A, 245, 650

Wang, J. M., 1994, AAnn Astropys.pJ, 434, 277 